\title{
Modelling the Influence of the Oxygen Concentration in the Gas Mixture (N2/O2) at Thermodynamic Equilibrium
}

\author{
O. Zeggai \\ Research Unit of Materials and \\ Renewable Energies \\ (URMER), University Abou \\ Bakr Belkaïd, B.P. 119, \\ Tlemcen, Algeria
}

\author{
A. Ould-Abbas \\ Research Unit of Materials and \\ Renewable Energies \\ (URMER), University Abou \\ Bakr Belkaïd, B.P. 119, \\ Tlemcen, Algeria
}

\author{
H.Zeggai \\ Research Unit of Materials and \\ Renewable Energies \\ (URMER), University Abou \\ Bakr Belkaïd, B.P. 119, \\ Tlemcen, Algeria
}

\begin{abstract}
Knowledge of the chemical composition of plasma is required for calculations and modeling in thermal plasmas. Indeed, from the knowledge of this composition can be calculated thermodynamic properties, transport coefficients and the radiative properties of a plasma environment. In this work, we propose to study the influence of thermodynamic equilibrium concentration of oxygen in a gas mixture $\mathrm{N} 2 / \mathrm{O} 2$. Particularly we study the evolution of the species density in the plasma created according to the temperature and pressure variables for $\mathrm{O} 2$ mixtures. A thermodynamic equilibrium, when you want to take into account a large number of species, two main methods are usually employed, one is based on the law of mass action and the other on minimization of the Gibbs. We decided in our study to the law of mass action and the method of Newton-Raphson. The results show that when the plasma is in thermodynamic equilibrium densities of the different species present in plasma that are functions of temperature and pressure. They are not independent because they are bound by certain laws of thermodynamic equilibrium
\end{abstract}

\section{Keywords}

Plasma, heat transfer, law of mass action, thermodynamic equilibrium, $\mathrm{N} 2 / \mathrm{O} 2$

\section{INTRODUCTION}

Nowadays, the pollution of the atmosphere has become a major environmental issue due to the rapid growth of industrial and technological development that requires high energy consumption. In the case of fossil fuels, this necessarily leads to an increase of emissions by industry, automotive, housing, etc ... gaseous air pollutants such as volatile organic compounds (VOCs) and various oxides (NOx , SOx, ozone, etc ...).

In this work we are interested in modeling the chemical composition at thermodynamic equilibrium of a gas mixture $(\mathrm{N} 2 / \mathrm{O} 2)$. Thus the law of mass action (law Saha and Guldberg and Waage law) allows the thermodynamic equilibrium to determine the concentrations of different species $(\mathrm{N}, \mathrm{N} 2$, electrons). We analyze in particular the influence of the concentration of oxygen $(\mathrm{O} 2)(1,10,50,90$ and $99 \%$ of $(\mathrm{O} 2))$ and pressure $(0.01$ bar 1 bar -10 bar) on the evolution of density of the two species: N, N2, the mixture was subjected to a power ranging from 1000 to $20000 \mathrm{~K}$.

\section{MODELLING}

Plasmas are home to a large number of chemical reactions that can be written as:

$\sum_{j=1}^{N} v_{j}^{d} A_{j} \leftrightarrows \sum_{j=1}^{N} v_{j}^{i} A_{j}$
Where $\left(v_{j}^{i}, v_{j}^{d}\right)$ are the stoichiometric coefficients of the inverse and direct reactions, $\mathrm{N}$ is the number of chemical species present in the mixture $\left(A_{j}\right)$ is the symbol of the chemical species $(j)$.

If the external conditions the plasma (pressure, temperature) are kept constant, for example by atmospheric pressure and by the electric field, then the chemical equilibrium is reached when the state functions are invariable. Therefore, we can write for the free energy:

$\left(\sum_{i=1}^{N}\left(\frac{\partial G}{\partial N_{i}} d N_{i}\right)_{N_{j \neq 1}}\right)_{T_{s i, P}}=0$

$\left(d N_{i}\right)$ is the variation in the number of species (i). For reaction (1) we have

$d N_{i}=v_{i}$

By introducing the chemical potential, the relation (2) becomes:

$\sum_{i=1}^{N} u_{i} v_{i}=0$

We have seen that the chemical potential $\left(u_{i}\right)$ is expressed in terms of the chemical potential calculated at the reference pressure $(\mathrm{P} 0)$ :

- At thermal equilibrium:

$$
u_{i}=u_{i}^{0}+R T \ln \frac{R T n_{i}}{p^{0}}
$$

- . With a thermal disequilibrium:

$$
\begin{aligned}
& \prod_{i}\left(n_{i}\right)^{R T_{t r i} v_{i}}=
\end{aligned}
$$

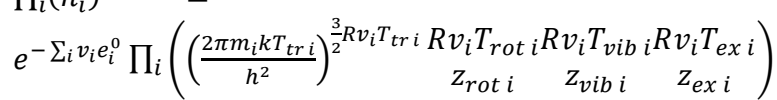

We note that the thermal equilibrium we find the formula (7). These two relationships are the mass action laws governing the balance of the constituents of a chemical reaction in thermal equilibrium and thermal non-equilibrium.

If we apply the formula (8) to the ionization

$\mathrm{A}^{+}+\mathrm{e}^{-} \rightleftarrows \mathrm{A}$

We find the law of Saha

$$
\frac{n_{A}+n_{e^{-}}}{n_{a}}=2\left(\frac{2 \pi m_{e^{-k T}}}{h^{2}}\right)^{\frac{3}{2}} \frac{Z_{\text {int } A^{+}}}{z_{\text {int } A}} \exp \left(\frac{-E_{i}}{R T}\right)
$$

Where $\left(E_{\mathrm{i}}\right)$ is the ionization energy

Similarly, if we apply the formula (8) to the dissociation of the type:

$\mathrm{A}+\mathrm{B} \rightleftarrows \mathrm{AB}$ 
We find the law of Guldberg and Waage

$$
\frac{n_{A}+n_{A}}{n_{A B}}=\left(\frac{2 \pi k T}{h^{2}} \frac{m_{A} m_{B}}{m_{A B}}\right)^{\frac{3}{2}} \frac{z_{\text {int } A} Z_{\text {int }} B}{z_{\text {int } A B}} \exp \left(\frac{-E_{d}}{R T}\right)
$$

Where $\left(E_{d}\right)$ is the dissociation energy of the molecule $A B$. The law of mass action out of thermal equilibrium is similar to that established by Potapov [1]. We find, asking the same assumptions internal temperature relationships used by many authors [2. 3, 4] under the name amended Saha equation for calculation of composition. In this case assumptions about internal temperatures are:

$\int \mathrm{T}_{\mathrm{vib}}=\mathrm{T}_{\mathrm{rot}}=\mathrm{T}_{\text {ex diat }}=\mathrm{T}_{\text {tr } \mathrm{l}}=\mathrm{T}_{1}$

$\left\{T_{\text {tre }}-=\mathrm{T}_{\text {ex at }}=\theta \mathrm{T}_{\text {tr } \mathrm{l}}=T_{e^{-}}\right.$

Under these conditions, we are led to the following relationship:

- For the ionization of atomic species:

$$
\begin{gathered}
n_{e^{-}}\left(\frac{n_{A^{+}}}{n_{A}}\right)^{\frac{1}{\theta}}=2\left(\frac{2 \pi m_{e^{-} k T_{e}}}{h^{2}}\right)^{\frac{3}{2}} \frac{z_{\text {int } A^{+}}\left(T_{e}-\right)}{z_{\text {int }}\left(T_{e^{-}}\right)} \exp \left(\frac{-E_{i}}{R T_{e^{-}}}\right)(13) \\
\bullet \quad \text { For the ionization of polyatomic species: } \\
n_{e^{-}}\left(\frac{n_{B^{+}}}{n_{B}}\right)^{\frac{1}{\theta}}=2\left(\frac{2 \pi m_{e^{-} k T_{e}}}{h^{2}}\right)^{\frac{3}{2}} \frac{z_{\text {int } B^{+}}\left(T_{l}\right)}{z_{\text {int } B}\left(T_{l}\right)} \exp \left(\frac{-E_{i}}{R T_{e^{-}}}\right) \text {(14) } \\
\bullet \quad \text { For the dissociation of a diatomic species: } \\
\frac{n_{A}+n_{A}}{n_{A B}}=\left(\frac{2 \pi k T}{h^{2}} \frac{m_{A} m_{B}}{m_{A B}}\right)^{\frac{3}{2}} \frac{\left(z_{\text {int } A}\left(T_{e^{-}}\right) z_{\text {int } B}\left(T_{e^{-}}\right)\right)^{\theta}}{z_{\text {int } A B}\left(T_{l}\right)} \exp \left(\frac{-E_{d}}{R T_{l}}\right) \\
(15)
\end{gathered}
$$

Solving this system of equations (chosen according to the case study) is to find concentrations that make the derivative of the free energy zero for each chemical reaction, which is to find the minimum of the free energy for each reaction.

The resolution of this non-linear system of equations was performed by the method of Newton - Raphson. We linearized thereof limited by a Taylor series expansion

\section{RESULTS AND DISCUSSION}

In this work we analyze in particular the influence of the concentration of oxygen $(\mathrm{O} 2)(1,10,50,90$ and $99 \% \mathrm{O} 2)$ and pressure ( 0.01 bar - 0.1 bar - 1 bar - 5 bar and 10 bar) on the change in density of eight species: N, N2. The mixture was subjected to a power ranging from 1000 to $20000 \mathrm{~K}$.

In Figures .1. For 6 we have shown the development of species density N, N2 as a function of temperature (1000 $20000 \mathrm{~K})$ for several different concentrations of oxygen $(\mathrm{O} 2)$ $(1,10,50,90$ and $99 \%)$ and three variable pressures $(0.01$ atm, $1 \mathrm{~atm}$, and 10ATM). The pressure is kept constant for every case of density analysis.

\subsection{Case for 0.01atm pressure}

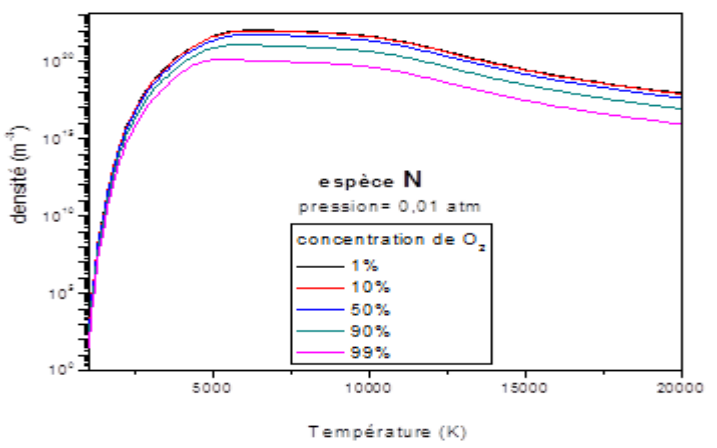

Fig.1 Evolution of species density $\mathrm{N}$ depending on the temperature for several different concentrations of oxygen $(\mathrm{O2})(1,10,50,90$ and $99 \%)$ and a constant pressure 0.01 atm

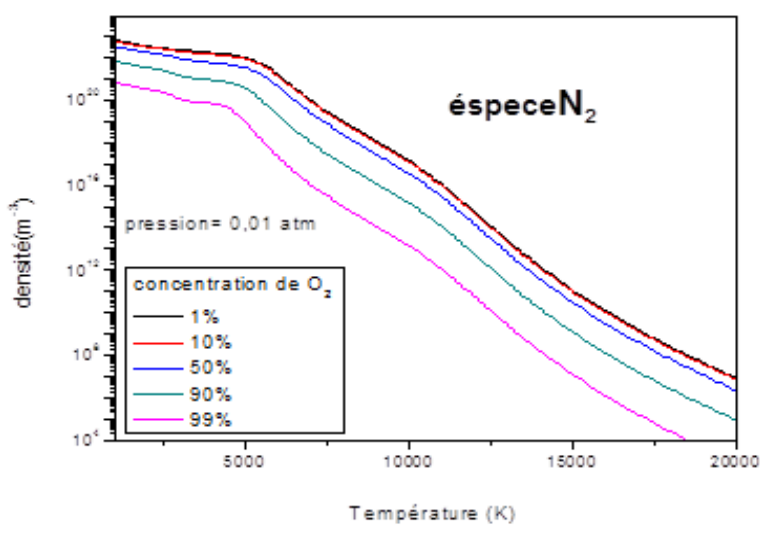

Fig .2 Evolution of species density $\mathrm{N}_{2}$ depending on the temperature for several different concentrations of oxygen $(\mathrm{O2})(1,10,50,90$ and $99 \%)$ and a constant pressure 0.01 atm

The Figures. 1 and 2 represents the change in the density of the species N, N2 based on the temperature (1000-20000 K) for several different concentrations of oxygen $(\mathrm{O} 2)(1,10,50,90$ and $99 \%$ ) and a constant pressure $0.01 \mathrm{~atm}$.

We note that for the $\mathrm{N}$ case the difference between the concentrations $1 \%$ and $99 \%$ is larger than $50 \%$ and $1 \%$. We also observe that the growth of nitrogen is very fast between 1000 and $5000 \mathrm{~K}$. This growth is due to the dissociation of the nitrogen molecule

$\mathrm{N}_{2}: \mathrm{e}+\mathrm{N}_{2} \rightarrow \mathrm{N}+\mathrm{N}+\mathrm{e}$

For temperatures above $5000 \mathrm{~K}$ density stabilizes to $12000 \mathrm{~K}$ because of reverse reactions, then it gradually decreases until 20000K because the $\mathrm{N}$ atom enters the establishment of other species $\left(\mathrm{N}^{+},\left(\mathrm{N}_{2}\right)^{+} \ldots\right)$ this all concentrations.

For the species $\mathrm{N}_{2}$ we observe a general decrease throughout the simulation of this species. Firstly, the first decline is slow and happens between 5000K (concentration 99\%) and 7000K (concentration 99\%) is due to the dissociation of the nitrogen molecule. Then the second reduction happens beyond $7000 \mathrm{~K}$ is faster than the first. It is due to several reactions creations of other species such as $\left(\mathrm{N}_{2}\right)^{+}, \mathrm{NO}, \mathrm{N}_{2} \mathrm{O}$. We observe a difference between the different concentrations begins to be significant from $50 \%$. 


\subsection{Case for 1 atm pressure}

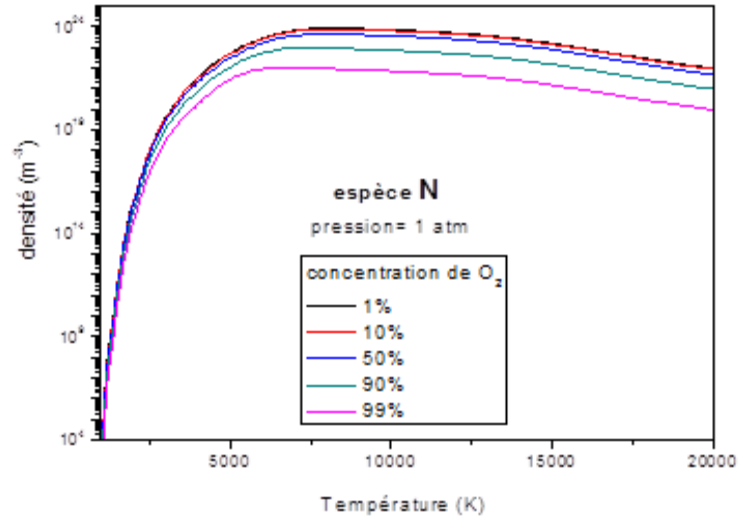

Fig 3. Evolution of species density $\mathbf{N}$ depending on the temperature for several different concentrations of oxygen (O2) $(1,10,50,90$ and $99 \%)$ and a constant pressure 1atm

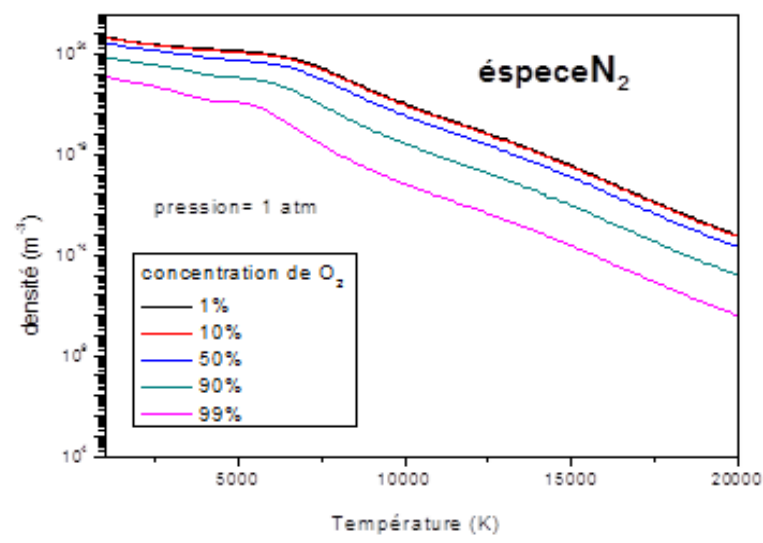

Fig 4. Evolution of species density $\mathbf{N} 2$ depending on the temperature for several different concentrations of oxygen (O2) $(1,10,50,90$ and $99 \%)$ and a constant pressure 1atm

We have shown in Figures 3 and 4, the evolution of the density of the same species as above, but this time for a constant pressure $1 \mathrm{~atm}$. We note in general as for Figures 1 the same rate of change of density, but the values increase with respect to those corresponding to $0.01 \mathrm{~atm}$.

\subsection{Case for $10 \mathrm{~atm}$ pressure}

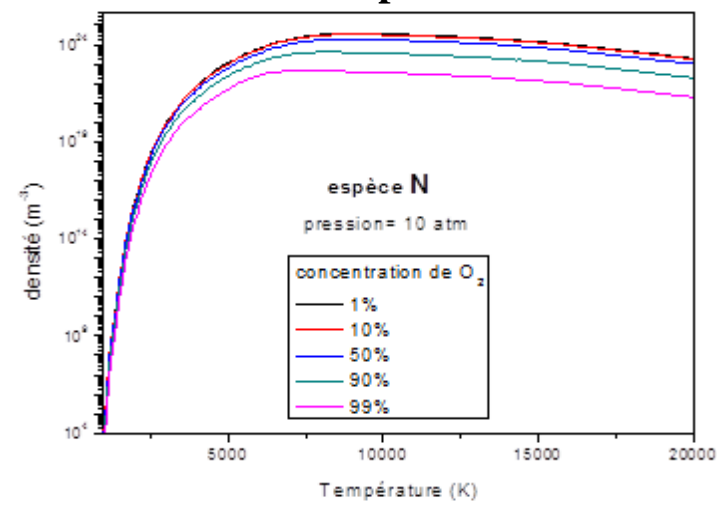

Fig 5. Evolution of species density $N$ depending on the temperature for several different concentrations of oxygen $\left(\mathrm{O}_{2}\right)(1,10,50,90$ and $99 \%)$ and a constant pressure 10 atm

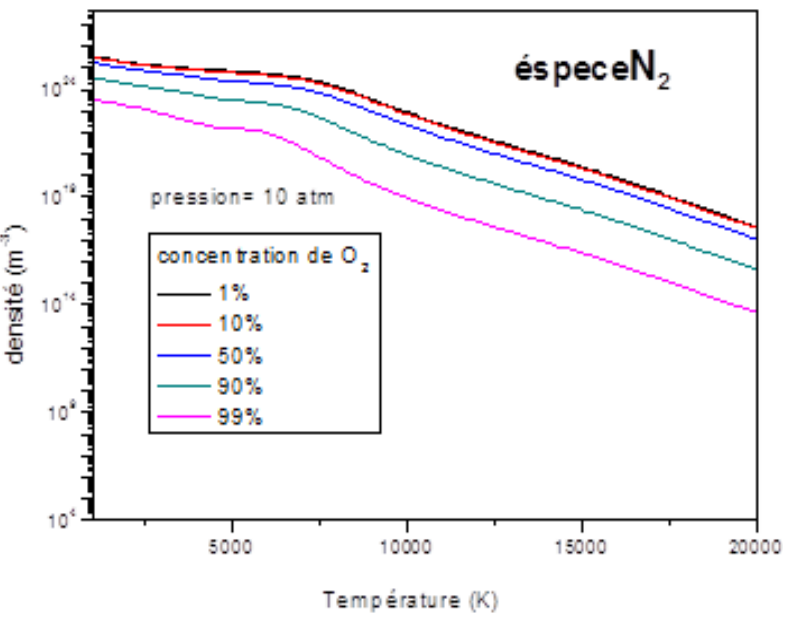

Fig 6. Evolution of species density $\mathrm{N}_{2}$ depending on the temperature for several different concentrations of oxygen (O2) $(1,10,50,90$ and $99 \%)$ and a constant pressure 10 atm

We conclude our analysis of the evolution of the density of the same species as above with the case of applying pressure $(10 \mathrm{~atm})$. The curves are shown in Figures 5 and 6 . Again, we observe the same behavior of density variation, always with an increase compared to the previous values, because of the gas law.

\section{CONCLUSION}

The chemical reactions in such a plasma are numerous and complex. Nevertheless, we can explain the movement of ionization and dissociation reactions by the ideal gas law. Indeed, for a given temperature, the pressure increases the number of particles per unit volume increases.

Analysis of these graphs shows that the influence of the concentration of the chemical species composition is very important for all species, especially for high pressures and high temperatures. However, it depends on each species for example, the influence of $\mathrm{N}$ radical concentration is significant from $15000 \mathrm{~K}$ while for other species it is significant for specific temperatures. This influence is different because of the energy of dissociation and ionization which are different for all of these species.

\section{REFERENCES}

[1] Potapov A.V « chemical equilibruim of multitemperature systems » High Temp, Vol.4, p 48-51, 1996

[2] Aubreton J. Labortoire de Matériaux Céramiques et traitement de Surface, Limoges Communication personnelle.

[3] Bonnefoi C. «contribution à l'étude des méthodes de résolution de l'équation de Boltzmann dans un plasma à deux températures: exemple de mélange Argonhydrogène », thèse d'état, 1983, Limoges

[4] Eymard M. these de $3^{\text {eme }}$ de cycle, $n^{\circ} 3101, \quad 1984$, Toulouse 